\title{
Lesões de pele ocasionadas por procedimento robótico versus aberto
}

RESUMO | Objetivo: identificar fatores associados a lesões de pele decorrentes de cirurgias urológicas robóticas versus convencionais em adultos/idosos. Método: revisão integrativa, etapas: Construção do protocolo de pesquisa; Formulação da pergunta - prática baseada em evidência, utilizando o acrônimo PICO; Definição dos descritores das estratégias de busca em cada uma das bases de dados selecionadas, que deviam ser variadas; Determinação, seleção e revisão dos critérios de inclusão e exclusão; Avaliação crítica dos estudos; Coleta de dados utilizando instrumentos que analisassem em pares; e Síntese dos resultados/dados agrupados por semelhança. Resultados: a estratégia de busca gerou 207 artigos. Resultando para análise final 7 artigos. Conclusão: são necessários novos estudos clínicos, que abordem os prejuízos e benefícios relacionados ao posicionamento cirúrgico robótico e abertos, direcionando assim, intervenções de enfermagem acuradas aos pacientes sob maior risco.

Palavras-chaves: Enfermagem Perioperatória; Procedimentos Cirúrgicos Robóticos; Urologia; Ferimentos e Lesões; Cuidados Intraoperatórios.

\begin{abstract}
Objective: to identify factors associated with skin lesions resulting from robotic versus conventional urological surgery in adults / elderly. Method: integrative review, stages: Construction of the research protocol; Formulation of the question - evidencebased practice, using the acronym PICO; Definition of search strategy descriptors in each of the selected databases, which should be varied; Determination, selection and review of inclusion and exclusion criteria; Critical evaluation of studies; Data collection using instruments that analyzed in pairs; and Summary of results / data grouped by similarity. Results: the search strategy generated 207 articles. Resulting in 7 articles for final analysis. Conclusion: further clinical studies are needed, addressing the losses and benefits related to robotic and open surgical positioning, thus directing accurate nursing interventions to patients at higher risk.
\end{abstract}

Keywords: Perioperative Nursing; Robotic Surgical Procedures; Urology; Wounds and Injuries; Intraoperative Care.

RESUMEN | Objetivo: identificar los factores asociados a las lesiones cutáneas resultantes de la cirugía urológica robótica versus convencional en adultos / ancianos. Método: revisión integradora, etapas: construcción del protocolo de investigación; Formulación de la pregunta - práctica basada en evidencia, utilizando el acrónimo PICO; Definición de descriptores de estrategias de búsqueda en cada una de las bases de datos seleccionadas, que deben ser variadas; Determinación, selección y revisión de criterios de inclusión y exclusión; Evaluación crítica de estudios; Recolección de datos utilizando instrumentos que se analizaron por parejas; y Resumen de resultados / datos agrupados por similitud. Resultados: la estrategia de búsqueda generó 207 artículos. Resultando en 7 artículos para el análisis final. Conclusión: se necesitan más estudios clínicos que aborden las pérdidas y beneficios relacionados con el posicionamiento quirúrgico robótico y abierto, dirigiendo así intervenciones de enfermería precisas a los pacientes de mayor riesgo.

Palabras claves: Enfermería Perioperatoria; Procedimientos quirúrgicos robóticos; Urología; Heridas y lesiones; Cuidados intraoperatorios.

\section{Beatriz Laureano de Souza}

Graduanda em Enfermagem/ da Escola de Enfermagem Aurora de Afonso Costa/ da Universidade Federal Fluminense/UFF. Niterói/RJ, Brasil.

ORCID: 0000-0002-1143-5240

\section{Maíra Danielle Gomes de Souza}

Doutora e Mestre em Cirurgia pela Universidade Federal de Pernambuco/ UFPE, Pós-graduanda enfermagem em cirurgia robótica/ Albert Einstein. Doutora e Mestre em Cirurgia pela Universidade Federal de Pernambuco/ UFPE, Recife, Brasil.

ORCID: 0000-0002-1814-0226

\section{Cecilia da Silva Moroni Primo}

Mestranda do Mestrado Profissional em Enfermagem Assistencial da Escola de Enfermagem Aurora de Afonso Costa/ da Universidade Federal Fluminense/UFF. Pós-graduada em enfermagem de Centro Cirúrgico, CME e RPA, Enfermeira HUCFF/UFRJ, Brasil. ORCID: 0000-0002-6423-1604
Natália Kaizer Rezende Ortega de Barros Enfermeira EEAAC/UFF, Residente em Centro Cirúrgico e CME/ Hospital Sírio-Libanês/ São Paulo/SP, Brasil.

ORCID: 0000-0001-5326-1237

\section{Thalita Gomes do Carmo}

Doutora em enfermagem, professora do Departamento de Enfermagem Médico Cirúrgico/MEM da Escola de Enfermagem Aurora de Afonso Costa/ da Universidade Federal Fluminense/UFF, Niterói/RJ, Brasil. ORCID: 0000-0002-5868-667X

Allanna da Costa Moura
Graduanda em Enfermagem/ da Escola de
Enfermagem Aurora de Afonso Costa/ da
Universidade Federal Fluminense/UFF. Ni-
terói/RJ, Brasil.
ORCID: 0000-0001-6300-631X

Recebido em: 29/01/2021

Aprovado em: 20/04/2021
INTRODUÇÃO

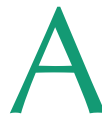
s cirurgias minimamente invasivas, ao contrário das cirurgias convencionais, são aquelas realizadas com reduções no tamanho das incisões, dor pós-operatória, sangramento e resposta inflamatória. Existem diferentes procedimentos considerados minimamente invasivos, como a videolaparoscopia, além da cirurgia robótica, que ganhou espaço em grande parte das especialidades cirúrgicas. ${ }^{1-2}$

Em 2000, o uso da robótica em tratamentos médicos atingiu um crescimento vertiginoso, devido ao desenvolvimento do sistema robótico Da Vinci. ${ }^{3}$ Essa plataforma apresenta três componentes principais: console (local onde o médico executa o procedimento); carrinho do paciente (4 braços robóticos, 1 para câmera 
endoscópica e 3 para os instrumentais cirúrgicos); e o carro visão. ${ }^{2-4}$

A cirurgia robótica no campo da urologia tem sido usada em muitos tratamentos cirúrgicos, principalmente na nefrectomia, cistectomia e prostatectomia. Nos últimos 20 anos, houve um aumento considerável na incidência do câncer de próstata na maioria dos países, além do câncer de bexiga que é um tumor maligno com uma alta taxa de invasividade e é um dos tipos mais comuns de câncer. ${ }^{5-7}$

No centro cirúrgico o enfermeiro realiza atividades específicas, sendo responsável por organizar e assegurar uma assistência segura ao paciente cirúrgico, utilizando a Sistematização da Assistência de Enfermagem Perioperatória (SAEP). ${ }^{8} \mathrm{O}$ processo de enfermagem realizado por meio da SAEP, trata-se de uma atividade imprescindível do enfermeiro para individualizar o cuidado, propiciando assim a promoção, manutenção e recuperação da saúde do paciente. ${ }^{9}$

Para iniciar um programa de cirurgia robótica, os funcionários bem treinados são de suma importância, e para isto, o enfermeiro tem como competência a formação e atualização de sua equipe. ${ }^{10} \mathrm{O}$ profissional de enfermagem deve garantir o melhor suporte possível, tanto a equipe quanto ao paciente. ${ }^{11}$ Para garantir a segurança do paciente durante o intraoperatório, deve-se estar atento ao posicionamento cirúrgico, e a imobilização do paciente, minimizando eventos adversos como lesão por pressão. ${ }^{10,12}$

O presente estudo busca identificar os fatores associados a lesões de pele decorrentes de cirurgias robóticas quan- do comparadas com as cirurgias convencionais na urologia em adultos e idosos, visando a melhoria na assistência prestada pelos enfermeiros atuantes na área.

\section{MÉTODO}

Revisão integrativa: 1.construção do protocolo de pesquisa; 2.formulação da pergunta dentro da prática baseada em evidência (PBE), utilizando o acrônimo PICO; 3.definição dos descritores das estratégias de busca em cada uma das bases de dados selecionadas pelo pesquisador; 4.determinação, seleção e dos critérios de inclusão e exclusão; 5.avaliação crítica dos estudos; 6.coleta de dados utilizando instrumentos de análise em pares; e 7.síntese dos resultados/dados agrupados por semelhança.

\section{Critérios de elegibilidade}

Pergunta do estudo: Quais são os fatores associados a lesões de pele decorrentes de cirurgias robóticas comparada com as cirurgias convencionais na urologia em adultos e idosos?

Critérios de inclusão: estudos com adultos maiores de 18 anos, comparativo das lesões de pele decorrentes de cirurgias robóticas versus convencional/ aberta; delineamento observacional, experimental ou quase experimental, e estudos com ou sem randomização. Excluiu-se estudos de fonte secundária, séries temporais ou caso controle, sem determinação de metodologia clara, teses e dissertações. Como filtros foram aplicados estudos em inglês, espanhol ou português; sem corte temporal.
Fontes de informação

A definição de descritores controlados foi referenciada a partir dos seguintes tesauros: Descritores em Ciências da Saúde (DeCS), MESH (Medical Subject Headings) e das palavras-chave do estudo. Os descritores controlados citados abaixo foram usados considerando os operadores booleanos "AND" e "OR" para pesquisa.

Devido às características específicas de cada base de dados, as estratégias de busca foram adaptadas de acordo com os objetivos e critérios de inclusão deste estudo. A busca dos artigos ocorreu em maio de 2020, e atualizada em junho de 2020, conforme Quadro 1.

\section{Busca e Seleção dos estudos}

A busca foi realizada nas seguintes bases de dados: CINAHL (Culmulative Index to Nursing and Allied Health Literature); PuBMed (Recurso de busca fornecido pela National Center for Biotechnology Information) e EMBASE (editora Elsevier) via Portal CAPES. Durante a seleção de fontes de evidência, o estudo foi dividido em dois momentos $\left(1^{\circ}\right.$ Busca nas bases de dados e inserção em planilha; $2^{\circ}$ Planilha foi encaminhada para 2 revisores independentes, que avaliaram os estudos em pares, de modo cego, aplicando os critérios de elegibilidade).

Coleta de dados e Síntese dos resultados

Seleção dos textos e preenchimento de uma planilha Excel construída com base no protocolo de pesquisa elaborado pelos autores, para a obtenção das informações necessárias para análise, consi-

Quadro 1.Estratégia PICO. Brasil, 2020.

\begin{tabular}{|l|c|c|c|} 
& Palavras-Chave & MESH & DECS \\
\hline População(and) & Adulto ou Idoso & Adult/Aged & Adulto/ldoso \\
\hline Interesse (and) & $\begin{array}{c}\text { Cirurgias urológicas robóticas/Período } \\
\text { Intraoperatória }\end{array}$ & $\begin{array}{c}\text { Robotic Surgical Procedures/Urology } \\
\text { Comparação (and) }\end{array}$ & $\begin{array}{c}\text { Procedimentos Cirúrgicos } \\
\text { Robóticos/Urologia }\end{array}$ \\
\hline Outcome (Desfecho) & Cirurgia convencional/Aberta & General surgery & Cirurgia geral \\
\hline Fonte: Própria autora. & & Wounds and Injuries & Ferimentos e lesões \\
\hline
\end{tabular}


Figura 1. Fluxograma do processo de seleção e inclusão do estudo segundo o Preferred

Reporting Items for Systematic Reviews and Meta-Analyses (PRISMA 2009). Niterói, Rio de Janeiro, 2020.

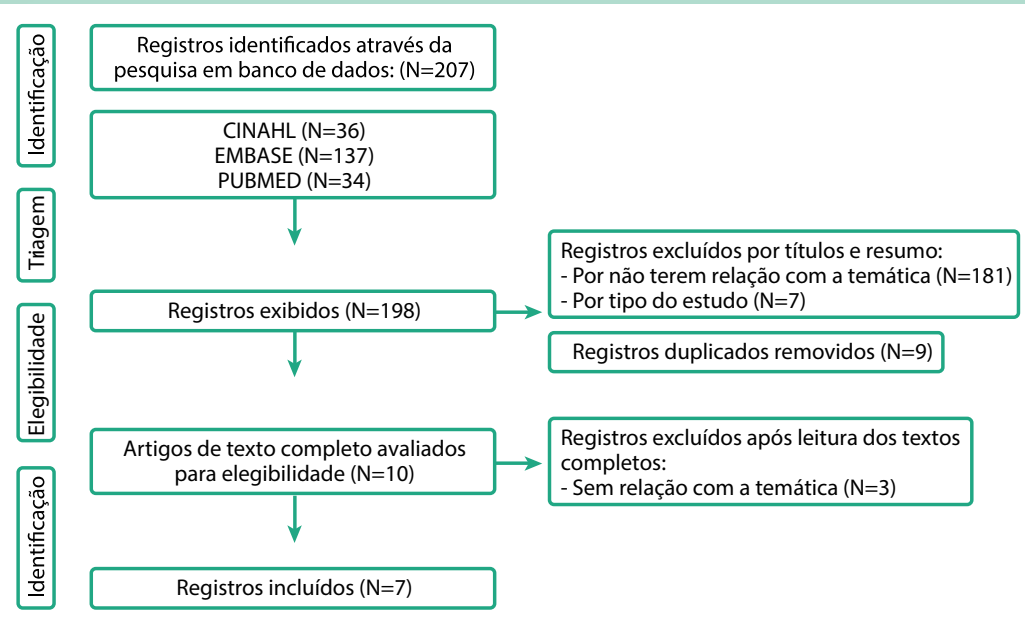

derando a pergunta do estudo e identificando quais são os fatores associados a lesões de pele decorrentes de cirurgias robóticas comparada com as cirurgias convencionais na urologia.

\section{RESULTADOS}

A Figura 01 detalha o fluxograma de seleção dos artigos.

No Quadro 2 são apresentados os 07 artigos incluídos no estudo.

Todos os estudos analisados realizaram um comparativo entre as técnicas cirúrgicas robótica e aberta nas cirurgias urológicas, destacam-se os prós e contras de cada uma dessas técnicas, no que se refere a: taxas de complicação, tempo de internação, sobrevida em até 90 dias e qualidade da recuperação no pós-operatório.

Quadro 2. Principais desfechos e referências dos estudos incluídos. Niterói, Rio de Janeiro, Brasil, 2020.

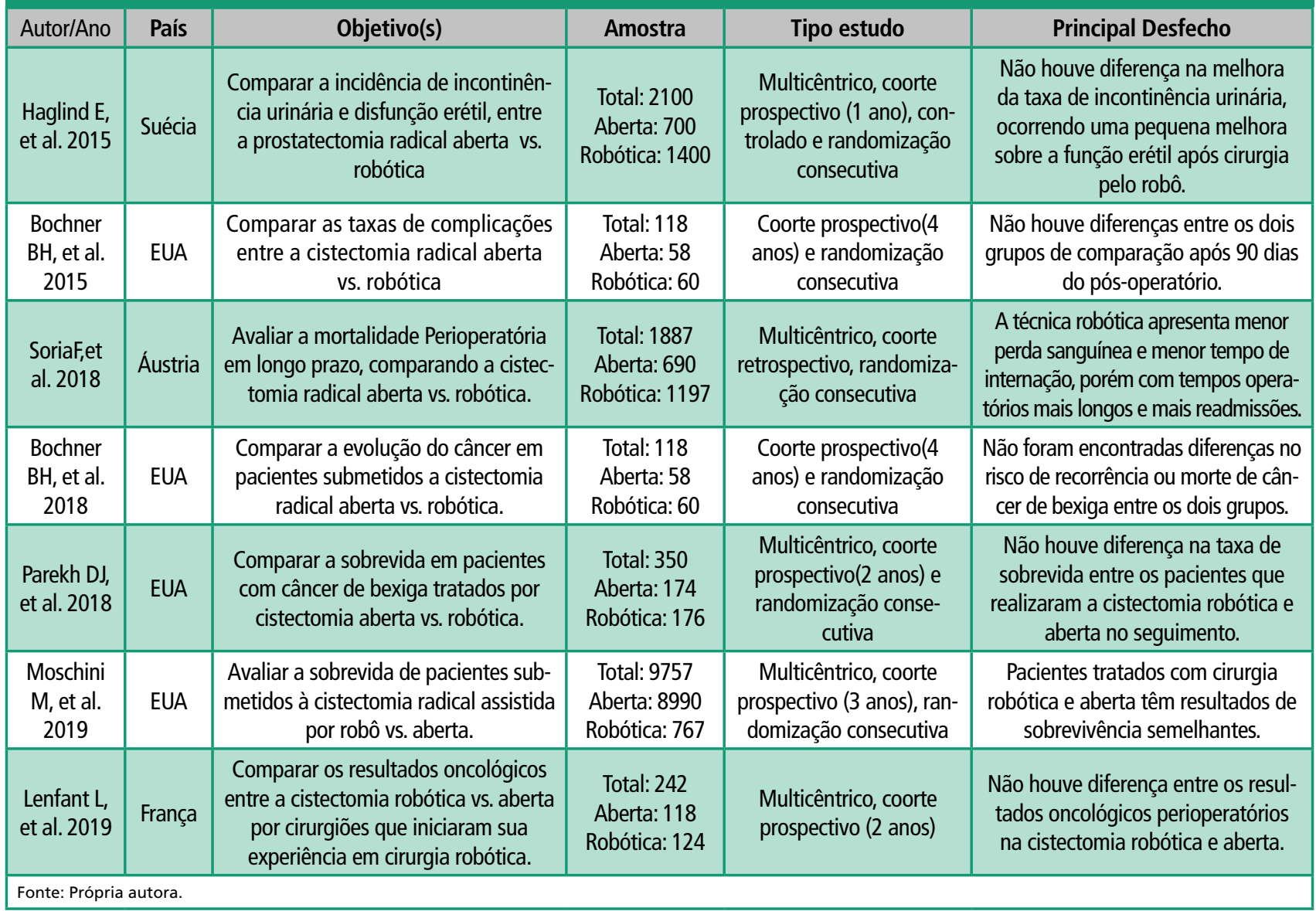


DISCUSSÃO

A maioria dos artigos incluídos apresentaram mais benefícios na técnica da cirurgia robótica comparados a cirurgia aberta, demonstrando a relevância dessa técnica para os pacientes com menor tempo de internação e perda sanguínea, menos complicações intra e pós-operatórias e preservação da função erétil. Três estudos demostraram que não houve diferença significativa na taxa de sobrevida dos pacientes. Enquanto dois artigos evidenciaram a necessidade de mais estudos para se detectar a superioridade da cirurgia robótica.

Em um estudo prospectivo, controlado, não randomizado comparando pacientes submetidos à prostatectomia por técnica assistida por robô e técnica aberta em 14 centros na Suécia foram elegíveis para a pesquisa 2625 homens e, após 12 meses, $21,3 \%$ que realizaram a cirurgia por robô e 20,2\% que realizaram a cirurgia aberta estavam com incontinência urinária. Após a prostatectomia robótica $70,4 \%$ comparado a $74,7 \%$ após prostatectomia aberta, apresentou disfunção erétil. Houve uma sutil melhora sobre a função erétil após operação por robô. ${ }^{13}$

Sooriakumaran et al. demonstrou que a cirurgia por via robótica favorece o melhor reconhecimento dos nervos planos de preservação durante a prostatectomia radical, desta forma, auxilia a preservação do feixe neurovascular e erétil. Ainda segundo o estudo, a recuperação da função erétil foi mais alta no grupo que realizou a cirurgia robótica entre 12 e 24 meses de acompanhamento, entretanto, em pacientes com tumores de alto risco, a recuperação da função erétil após 24 meses foi maior no grupo que realizou a cirurgia aberta. ${ }^{14}$

O programa de ensino domiciliar após a alta hospitalar constitui-se de uma intervenção educacional realizada por meio da combinação de aconselhamento oral, escrito e por telefone que buscou incentivar os pacientes em seu desenvolvimento sobre o autocuidado e a diminuição de morbidade psicológica. Esse estudo mostrou-se clinicamente eficaz para a intervenção proposta demonstrando a importância do profissional de enfermagem na preparação dos pacientes para a alta hospitalar e cuidados pós-operatórios no tratamento de neoplasias através de estratégias educacionais. ${ }^{15}$

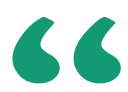

Ainda com relação a taxa de sobrevida, um estudo multicêntrico prospectivo observou que há desvantagens com relação ao tempo de realização do procedimento (curva de aprendizagem), custo e não há diferença significativa quanto a taxa de sobrevida do câncer quando comparamos a cirurgia aberta e a
robótica.

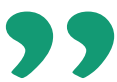

O estudo de Khan et al. comparou as três técnicas cirúrgicas demonstrando que o tempo cirúrgico foi significativamente maior na cirurgia robótica e que não houve diferenças significativas nas medidas de qualidade de vida analisadas, entretanto, o estudo apresentou algumas limitações como o tamanho amostral reduzido e viés do cirurgião. ${ }^{16}$ Nesse contexto, um estudo com 118 pacientes designados aleatoriamente para serem submetidos a cistectomia radical mais linfadenectomia pélvica (60 por técnica robótica e 58 por técnica aberta), não encontrou diferenças no risco de recorrência ou morte de câncer de bexiga entre os dois grupos. ${ }^{17}$

A técnica de cirurgia robótica foi associada com uma menor perda de sangue no intraoperatório, porém com um tempo cirúrgico aumentado, facilitando o surgimento de lesões na pele do paciente. O estudo também evidenciou que não houve diferenças significativas quanto ao tempo de internação hospitalar ou taxas de complicações em ambas as técnicas cirúrgicas. ${ }^{18}$

Ainda com relação a taxa de sobrevida, um estudo multicêntrico prospectivo observou que há desvantagens com relação ao tempo de realização do procedimento (curva de aprendizagem), custo e não há diferença significativa quanto a taxa de sobrevida do câncer quando comparamos a cirurgia aberta e a robótica. ${ }^{19}$ Comparativamente, Soria et al. demonstrou que, a cistectomia radical robótica apresentou menor perda sanguínea e menor tempo de internação, e tempos operatórios mais longos. ${ }^{20}$ Moschini et al. demonstrou que, a cirurgia robótica apresenta muitos benefícios à curto prazo, como: diminuição da perda sanguínea e tempo de internação, entretanto, não houve diferença significativa quanto a taxa de sobrevida do câncer ao comparar a cirurgia aberta e a robótica, sugerindo a realização de mais estudos prospectivos. $^{21}$

A cirurgia robótica mesmo sendo um método eficaz de técnica cirúrgica, apresenta desvantagens onerosas, atualmente os custos para aquisição do Da Vinci Surgical System ${ }^{\circledR}$ variam de US\$ 1 milhão a US\$ 2,5 milhões por unidade, além de reformas para adequação do ambiente físico e manutenções do sistema gerando 
um alto custo para o seu funcionamento. ${ }^{11}$

Existe uma lacuna do conhecimento que foi detectada durante a busca dos artigos, uma vez que não foram recuperados estudos que abordassem uma comparação entre as técnicas robótica e aberta, com relação a lesões de pele pelo posicionamento intraoperatório. Uma vez que, os procedimentos robóticos por terem uma maior duração, podem gerar mais danos a pele e tecidos dos pacientes. ${ }^{22}$ Sugere-se assim, mais estudos sobre os potenciais riscos a pele do paciente, constitui uma limitação do estudo, a não realização da busca de artigos em outras bases de dados.

\section{CONCLUSÃO}

Existe um maior benefício da cirurgia robótica em relação a convencional em prostatectomias radiais, com preservação da função erétil, já na cistectomia há menor sangramento, e até menor tempo de in- ternação. Não foram encontrados estudos que comparassem os fatores relacionados a incidência de lesões de pele entre os pacientes submetidos a cirurgia robótica e aqueles que realizam o tratamento convencional da cirurgia aberta. Logo, conclui-se que são necessários novos estudos clínicos, que abordem os prejuízos e benefícios relacionados ao posicionamento cirúrgico robótico e abertos, direcionando assim, intervenções de enfermagem acuradas aos pacientes sob maior risco.

\section{Referências}

1. Mariani AW, Pêgo-Fernandes PM. Minimally invasive surgery: a concept already incorporated. Sao Paulo Med. J., São Paulo, v. 131, n. 2, p. 69-70,2013.[acesso em 2020Jun 10]. DOI: https://doi.org/10.1590/S151631802013000100015

2. Veiga, DNMFR. Cirurgia minimamente invasiva-sistema Da Vinci-cirurgia roboticamente assistida. [tese]. Porto (PT): Instituto de Ciências Biomédicas de Abel Salazar da Universidade do Porto; 2011.

3. Cem®ah, MD. Does Robot-assisted Surgery in Urology Has Benefits? The Current Status. Bull Urooncol, v. 18, p. 117-119, 2019.DOI: https://doi. org/10.4274/uob.galenos.2019.1202

4. Pitasse $C$ et al. A Cirurgia Robótica nas Organizações Públicas de Saúde: 0 Caso do Instituto Nacional de Câncer (INCA). Administração Pública e Gestão Social, v. 8, n. 3. 2016. Disponível em: http://www.apgs.ufv.br

5. Wang $Y$, Gieschen $H$, Greenberger $M$ et al. Survival After Robotic-Assisted Prostatectomy for Localized Prostate Cancer: An Epidemiologic Study. Annals of Surgery. 2019 Oct. [acesso em 2020 Jun 12]. Doi: https://doi.org/10.1097/ SLA.0000000000003637

6. Cao L, Yang Z, Qi L, Chen M. Robot-assisted and laparoscopic vs open radical prostatectomy in clinically localized prostate cancer: perioperative, functional, and oncological outcomes: a systematic review and meta-analysis. Medicine. 2019; v. 98, n22. [acesso em 2020 Jun 12]. Disponível em: https:// www.ncbi.nlm.nih.gov/pmc/articles/PMC6709105/

7. Junior JRC, Andreoni C, Lemos GC, da Fonseca Filho LL, Di Pietro DL, Pinto W, Neto MC. Resultados iniciais da prostatectomia radical robô-assistida no Brasil. Rev. Einstein. 2018; 7(4), 488-493. [acesso em 2020 Jun 12]. Disponível em: http://apps.einstein.br/revista/arquivos/PDF/1294-Einsteinv7n4p488-93_ port.pdf

8. Jost MT et al. Sistematização da assistência de enfermagem perioperatória: avaliando os processos de trabalho no transoperatório. Enfermagem em Foco. 2020 Fev. v. 10, n. 7. [acesso em 2020 Jun 11]. Disponível em: http://revista. cofen.gov.br/index.php/enfermagem/article/view/2354/548.

9. Prearo M, Fontes CMB. Sistematização da assistência de enfermagem na sala de recuperação pós anestésica: revisão integrativa. Enfermagem em Foco. 2020 Fev. v. 10, n. 7. [acesso em 2020Jun 11]. Disponível em: http://revista. cofen.gov.br/index.php/enfermagem/article/view/2470/562.

10. Peng L, Li J, Cao D, Ren Z, Wei T, You C, Li Y. Can robotic-assisted radical cystectomy provide patients with a smaller trauma and faster recovery period? A systematic review and meta-analysis of comparative trials. Journal of Cancer Research and Clinical Oncology. 2020; p. 1-11. [acesso em 2020 Jun 12]. Disponivel em: https://link.springer.com/article/10.1007/s00432-020-03183-0 11. Pinto $E V$, Lunardi $L S$, Treviso $P$, Botene DZDA. Atuação do enfermeiro na cirurgia robótica: desafios e perspectivas. Rev. SOBECC. 2018; v. 23, n.1. [acesso em 2020 Jun 12]. Disponível em: http://brutus.facol.com/plataforma/assets/ uploads/base/publicados/4fe664f61075e001 cf78cb9e4bce29a8.pdf 12. Ferreira F, dos Santos PF, Dalto APP, da Silva Granandeiro D, de Azeredo
Granadeiro RM, de Melo NGS, Passos JP. Autonomia e gerenciamento do enfermeiro no serviço de cirurgia robótica. Saúde Coletiva (Barueri). 2019; n. 51, p.1954-1958. [acesso em 202013 Jun]. Disponível em: http://revistas.mpmcomunicacao.com.br/index.php/saudecoletiva/article/view/182

13. Haglind $\mathrm{E}$ et al. Urinary incontinence and erectile dysfunction after robotic versus open radical prostatectomy: a prospective, controlled, non randomised trial. EuropeanUrology. 2015;68(2):216-25. [acesso em 2020 Jul 07].Doi: https://doi.org/10.1016/j.eururo.2015.02.029.

14. Sooriakumaran P et al. Erectile Function and Oncologic Outcomes Following Open Retropubic and Robot-assisted Radical Prostatectomy: Results from the Laparoscopic Prostatectomy Robot Open Trial. European Urology. 2018;73(4):618-627.

15. da Mata LRFP, Bernardes MFVG, Azevedo C, Chianca TCM, Pereira MG, Carvalho EC. Jacobson and Truax Method: evaluation of the clinical effectiveness of a home care program after prostatectomy. Rev. Latino-Am. Enfermagem. 2018; v. 26. [acesso em 2020 Jun 14]. Disponivel em: https://www.scielo. br/scielo.php?pid=S0104-11692018000100319\&script=sci_arttext\&tlng=pt 16. Khan MS et al. A Single-centre Early Phase Randomised Controlled Threearm Trial of Open, Robotic, and Laparoscopic Radical Cystectomy (CORAL). EuropeanUrology. 2016; v. 69, n. 4. [acesso em 2020 Jun 16]. Doi:http://dx.doi. org/10.1016/j.eururo.2015.07.038

17. Bochner BH et al. Randomized Trial Comparing Open Radical Cystectomy and Robot-assisted Laparoscopic Radical Cystectomy: Oncologic Outcomes. European Urology. 2018 Oct;74(4):465-471. [acesso em 2020 Jul 05]. Doi: 10.1016/j.eururo.2018.04.030.

18. Satkunasivam R, Tallman CT, Taylor JM, MILES BJ, Klaassen Z, Wallis CJD. Robot-assisted Radical Cystectomy Versus Open Radical Cystectomy: a meta-analysis of oncologic, perioperative, and complication-related outcomes. European Urology Oncology. 2019 [S.L.], v. 2, n. 4. [acesso em 2020 Jul 10]. Doi: http://dx.doi.org/10.1016/j.euo.2018.10.008.

19. Parekh DJ et al. Robot-assisted radical cystectomy versus open radical cystectomy in patients with bladder cancer (RAZOR):an open-label, randomised, phase 3, non-inferiority trial. The Lancet. 2018; v. 391. [acesso em $2020 \mathrm{Jul} 10$ ]. Doi: http://dx.doi.org/ 10.1016/S0140-6736(18)30996-6.

20. Soria $\mathrm{F}$ et al. Comparative Effectiveness in Perioperative Outcomes of Robotic versus Open Radical Cystectomy: Results from a Multicenter Contemporary Retrospective Cohort Study. Eur Urol Focus. 2018. Doi:https://doi. org/10.1016/j.euf.2018.11.002.

21. Moschini M et al. Open Versus Robotic Cystectomy: a propensity score matched analysis comparing survival outcomes. Journal of Clinical Medicine. 2019; v. 8, n. 8. [acesso em 2020 Jul 08]. Doi:http://dx.doi.org/10.3390/ jcm8081192.

22. Angelo $\mathrm{C}$ da $\mathrm{S}$ et al. Efetividade do protocolo prevenção de lesões de pele em cirurgias urológicas robóticas. Revista Sobecc. 2017; v. 22, n. 3. [acesso em 2020 Jun 14]. Doi: http://dx.doi.org/10.5327/z1414-4425201700030006. 